\title{
Well-Posedness of Minimal Time Problems with Constant Dynamics in Banach Spaces *
}

\author{
Giovanni Colombo $^{\dagger} \quad$ Vladimir V. Goncharov ${ }^{\ddagger} \quad$ Boris S. Mordukhovich ${ }^{\S}$
}

\section{Dedicated to Jean-Baptiste Hiriart-Urruty in honor of his 60th birthday}

\begin{abstract}
This paper concerns the study of a general minimal time problem with a convex constant dynamics and a closed target set in Banach spaces. We pay the main attention to deriving sufficient conditions for the major well-posedness properties that include the existence and uniqueness of optimal solutions as well as certain regularity of the optimal value function with respect to state variables. Most of the results obtained are new even in finite-dimensional spaces. Our approach is based on advanced tools of variational analysis and generalized differentiation.
\end{abstract}

Keywords Minimal time function · Minimal time projection · Variational analysis · Generalized differentiation

Mathematical Subject Classifications (2000) 49J53 · 49J52 · 46B20 · 49J50

\section{Introduction}

A general class of minimal time problems with constant dynamics can be described as:

$$
\text { minimize } t>0 \text { subject to }(x+t F) \cap C \neq \emptyset, \quad x \in X,
$$

where $X$ is a Banach space of state variables, $C \subset X$ is a closed target set, and $F \subset X$ is a closed, convex, and bounded set with $0 \in \operatorname{int} F$. We call $F$ the constant dynamics reflecting the fact that the optimal value in problem (1.1) is the minimal time needed to attain the target set $C$ from $x$ by trajectories of the differential inclusion $\dot{x}(t) \in F$. The

${ }^{*}$ This work was partially supported by M.I.U.R., project "Viscosity, Metric, and Control Theoretic Methods for Nonlinear Partial Differential Equations" and by CARIPARO "Visiting Professors' Program."

${ }^{\dagger}$ Dipartimento di Matematica Pura e Applicata, Università di Padova, via Trieste 63, 35121 Padova, Italy; e-mail: colombo@math.unipd.it.

${ }^{\ddagger}$ Departamento de Matemática, Universidade de Évora, R. Romão Ramalho 59, P/7000/671 Évora, Portugal; e-mail: goncha@uevora.pt.

${ }^{\S}$ Department of Mathematics, Wayne State University, Detroit, MI 48202, USA; e-mail: boris@math.wayne.edu. Research of this author was partially supported by the US National Science Foundation under grant DMS-0603846 and by the Australian Research Council under grant DP-12092508. 
imposed requirements on the dynamics and target are our standing assumptions in this paper. Various properties of optimal solutions to (1.1) were studied in $[8,9,10,12,17]$ and the references therein in finite and infinite dimensions.

The major optimal characteristics of problem (1.1) are given by the optimal value function (known also as the minimal value/time function) defined by

$$
\left.T_{C}^{F}(x)=T(x):=\inf \{t>0 \mid(x+t F) \cap C) \neq \emptyset\right\}=\inf _{y \in C} \rho_{F}(y-x),
$$

where $\rho_{F}(\cdot)$ is the classical Minkowski functional/gauge,

$$
\rho_{F}(u):=\inf \left\{t>0 \mid t^{-1} u \in F\right\}, \quad u \in X,
$$

and by the generalized/minimal time projection

$$
\Pi_{C}^{F}(x):=\left\{y \in C \mid \rho_{F}(y-x)=T(x)\right\}, \quad x \in X,
$$

which is generally a set-valued mapping $\Pi: X \rightrightarrows C$ with possibly empty values.

Observe that the minimal value function (1.2) can be considered as a natural generalization of the classical distance function

$$
\operatorname{dist}(x ; C):=\inf _{y \in C}\|y-x\|, \quad x \in X,
$$

which corresponds to (1.2) with $F=\mathbb{B}$, the closed unit ball of the space in question. In the latter case, the generalized projection (1.4) reduces to the usual metric projection

$$
\Pi_{C}(x):=\{y \in C \mid \operatorname{dist}(x ; C)=\|y-x\|\}, \quad x \in X,
$$

of $x$ onto $C$ induced by the norm of the Banach space $X$ under consideration.

The main objective of this paper is to study well-posedness of the minimal time problem (1.1) in finite-dimensional and infinite-dimensional spaces. By well-posedness we understand here the existence and uniqueness of the generalized projection (1.4) in connection with certain regularity properties of the minimal time function (1.2). Since the latter function is intrinsically nonsmooth, our study strongly involves the usage of appropriate tools of advanced variational analysis and generalized differentiation.

Concerning most relevant results of the previous investigations, note that the proximal and Fréchet subdifferentials of the minimal time function were computed first in Hilbert spaces $[8,9]$. These results generalize the corresponding formulas obtained earlier in $[3,6,13]$ for the case of $F=\mathbb{B}$. Furthermore, based on these formulas and adapting respective arguments used earlier in $[4,6,7,19]$ to study the metric projection (1.6), the authors of [8,9] established some well-posedness properties (existence, uniqueness, and certain regularity near the target $C$ ) of the minimal time projection (1.4). The assumptions in $[8,9]$ involve both "external sphere type" conditions on $C$, called $\varphi$-convexity or proximal smoothness, and a kind of uniform strict convexity of the dynamics $F$. Quite recently [10], these conditions have been sharpened and localized by 
introducing a certain regular curvature of a convex body. Besides that, an alternative hypothesis involving the duality mapping, which requires neither $\varphi$-convexity of the target set $C$ nor rotundity/strict convexity of the dynamics $F$, is proposed in [10, Section 5]).

Formulas for evaluating the proximal and Fréchet subdifferentials of the minimal time function (1.2) in general Banach spaces, extending the corresponding results of [8,9] from the Hilbert space setting, are obtained in [12]. The most recent results in this direction derived in [17] provide tight upper estimates as well as exact formulas for computing the $\varepsilon$-subdifferentials of the Fréchet type and the limiting/Mordukhovich subdifferential of the minimal time function at both in-set $(\bar{x} \in C)$ and out-of-set $(\bar{x} \notin C)$ points in arbitrary Banach spaces $X$. The results of [17] extend those obtained in $[3,13,15,16]$ for the latter subdifferentials of the distance function (1.5). They are used in what follows to establish some regularity properties of the minimal time function (1.2).

In this paper we develop an approach to the existence and uniqueness of the minimal time projection (1.4) that is different from the previous investigations discussed above. Namely, we study the existence and uniqueness of (1.4) through subdifferentiability of the minimal time function (1.2) at a fixed point $\bar{x} \in X \backslash C$ (not necessarily in a neighborhood of the target). A prototype result for the metric projection mapping (1.6) can be found in [2, Lemma 6], which proves the existence and uniqueness of the metric projection of $\bar{x} \in X \backslash C$ onto $C$ provided that the distance function (1.5) is Fréchet subdifferentiable at $\bar{x}$ and that the space $X$ admits a Fréchet smooth renorm. We obtain a similar result in this direction for the general minimal time projection (1.4) by using some local rotundity properties of the dynamics $F$ expressed in terms of the duality mapping associated with $F$. We also derive other verifiable conditions, including necessary and sufficient ones, for the existence and uniqueness of the minimal time projection that are expressed in terms of the dynamics and target sets in (1.1).

Finally, we derive sufficient conditions ensuring the lower/subdifferential regularity of (1.2) at both in-set and out-of-set points of the target. The assumptions imposed and the results obtained are illustrated by examples and counterexamples in both finitedimensional and infinite-dimensional spaces.

The rest of the paper is organized as follows. Section 2 contains some definitions and preliminary material from convex and variational analysis widely used in the paper.

In Section 3 we establish relationships between the minimal time projection mapping (1.4) and its $\varepsilon$-enlargement, from one side, and the duality mapping for the dynamics and the Fréchet subdifferential of the minimal time function at the reference point from the other. This allows us to derive in Section 4 sufficient conditions for the existence and uniqueness of the minimal time projection via some rotundity properties of the dynamics. This section contains also some general observations concerning rotundity as well as a counterexample showing that problem (1.1) is no longer well posed if the rotundity condition is violated.

In Section 5, by using a somewhat different approach, we obtain characterizations of the existence and uniqueness of the minimal time projection in terms of the Gâteaux differentiability of the support function associated with an appropriate subset of the target $C$ in (1.1). Sufficient conditions of another type are derived under the Fréchet 
differentiability of the support function associated with the $\varepsilon$-enlargement of the minimal time projection onto $C$.

The final Section 6 provides conditions ensuring the lower regularity of the minimal time function (1.2) that imply, in the case of reflexive and more general Asplund spaces, the Fréchet subdifferentiability of (1.2) at the reference points, which is essentially used in the most interesting results of Sections 4 and 5 on the uniqueness of the minimal time projection (1.4). On the other hand, we present examples showing that the lower regularity of (1.2) is not necessary for the uniqueness of (1.4), while even Fréchet differentiability alone does not ensure the uniqueness of generalized projections.

\section{Basic Definitions and Preliminaries}

In this section we present some constructions and facts from convex and variational analysis needed in what follows. The reader can consult the books [18] in the convex case and [14] in the general setting for more details, discussions, and further references.

Unless otherwise stated, the space $X$ under consideration is arbitrary Banach with the norm $\|\cdot\|$, and the canonical pairing $\langle\cdot, \cdot\rangle$ between $X$ and its topological dual $X^{*}$. Given a nonempty set $F \subset X$, we recall the construction of its polar

$$
F^{\circ}:=\left\{x^{*} \in X^{*} \mid\left\langle x^{*}, x\right\rangle \leq 1 \text { for all } x \in F\right\},
$$

which is always a convex (even when $F$ is nonconvex) and weak* closed subset of the dual space $X^{*}$. The support function $\sigma_{F}: X^{*} \rightarrow \overline{\mathbb{R}}:=(-\infty, \infty]$ of $F$ is

$$
\sigma_{F}\left(x^{*}\right)=\sup _{x \in F}\left\langle x^{*}, x\right\rangle, \quad x^{*} \in X^{*} .
$$

Note that (2.1) is always convex and lower semicontinuous (l.s.c.) on $X^{*}$, and it is the Legendre-Fenchel conjugate of the indicator function $\delta_{F}(\cdot)$ of $F$ equal to 0 for $x \in F$ and $\infty$ otherwise.

The duality mapping $\mathfrak{J}_{F}: F^{\circ} \rightrightarrows F$ for $F$ is defined by

$$
\mathfrak{J}_{F}\left(x^{*}\right):=\left\{x \in F \mid\left\langle x^{*}, x\right\rangle=\sigma_{F}\left(x^{*}\right)\right\}, \quad x^{*} \in F^{\circ},
$$

which reduces to the simplified representation on the boundary bd $F^{\circ}$ of $F^{\circ}$ :

$$
\mathfrak{J}_{F}\left(x^{*}\right)=\left\{x \in F \mid\left\langle x^{*}, x\right\rangle=1\right\}, \quad x^{*} \in \operatorname{bd} F^{\circ} .
$$

Furthermore, in the case of reflexive spaces $X$ we have the relationship

$$
\partial \sigma_{F}\left(x^{*}\right)=\mathfrak{J}_{F}\left(x^{*}\right) \text { for all } x^{*} \in F^{\circ}
$$

where $\partial$ stands for the classical subdifferential of convex analysis. It is worth mentioning that, under our standing assumptions on the dynamics $F$, both the Minkowski gauge (1.3) and the minimal time function (1.2) are Lipschitz continuous on $X$. Furthermore,

$$
\rho_{F}(x)=\sigma_{F^{\circ}}(x) \text { and } \rho_{F^{\circ}}\left(x^{*}\right)=\sigma_{F}\left(x^{*}\right) \text { for all } x \in X, x^{*} \in X^{*} .
$$


Using (1.2) and (1.3) allows us to consider, together with the (possibly empty) minimal time projection (1.4), its always nonempty enlargement called $\varepsilon$-approximate minimal time projection as $\varepsilon>0$ and denoted by

$$
\Pi_{C}^{F}(x, \varepsilon):=\left\{y \in C \mid \rho_{F}(y-x) \leq T(x)+\varepsilon\right\} \neq \emptyset \text { for all } x \in X .
$$

Note that both the minimal time projection (1.4) and its $\varepsilon$-enlargement (2.5), for all $\varepsilon>0$, are closed subsets of $X$ under our standing assumptions.

Let us further recall two outer/upper limits of the Painlevé-Kuratowski type needed in this paper. Given a set-valued mapping $G: Y \rightrightarrows Z$ between two Banach spaces, consider the weak sequential outer limit of $G$ at $\bar{y}$ defined and denoted by

$$
\begin{aligned}
w-\operatorname{Limsup}_{y \rightarrow \bar{y}} G(y):=\{z \in Z \mid & \exists \text { sequences } y_{k} \rightarrow \bar{y} \text { and } z_{k} \stackrel{w}{\rightarrow} z, \text { as } k \rightarrow \infty \\
& \text { with } \left.z_{k} \in G\left(y_{k}\right) \text { for all } k \in \mathbb{N}:=\{1,2, \ldots\}\right\},
\end{aligned}
$$

where $\stackrel{w}{\rightarrow}$ signifies the convergence w.r.t. the weak topology on the image space $Z$.

Another version of the sequential Painlevé-Kuratowski outer limit needed below concerns set-valued mappings $G: X \rightrightarrows X^{*}$ between a Banach space $X$ and its topological dual. We say that the construction

$$
\begin{aligned}
w^{*}-\operatorname{Limsup}_{x \rightarrow \bar{x}} G(x):=\left\{x^{*} \in X^{*} \mid\right. & \exists \text { sequences } x_{k} \rightarrow \bar{x} \text { and } x_{k}^{*} \stackrel{w^{*}}{\rightarrow} x^{*} \\
& \text { with } \left.x_{k}^{*} \in G\left(x_{k}\right) \text { for all } k \in \mathbb{N}\right\}
\end{aligned}
$$

is the $w^{*}$-sequential outer limit of $G$ as $x \rightarrow \bar{x}$, where $w^{*}$ signifies the weak ${ }^{*}$ topology of the dual space $X^{*}$. Note that we use sequences in both constructions (2.6) and (2.7) although neither the weak topology of a Banach space nor the weak* topology of a dual Banach space is generally sequential.

Consider next an extended-real-valued function $\varphi: X \rightarrow \overline{\mathbb{R}}$ on a Banach space $X$. Given $\varepsilon \geq 0$, the $\varepsilon$-subdifferential of $\varphi$ at $\bar{x} \in \operatorname{dom} \varphi:=\{x \in X \mid \varphi(x)<\infty\}$ is defined by

$$
\widehat{\partial}_{\varepsilon} \varphi(\bar{x}):=\left\{x^{*} \in X^{*} \mid \liminf _{x \rightarrow \bar{x}} \frac{\varphi(x)-\varphi(\bar{x})-\left\langle x^{*}, x-\bar{x}\right\rangle}{\|x-\bar{x}\|} \geq-\varepsilon\right\}
$$

with $\widehat{\partial} \varphi(\bar{x}):=\widehat{\partial}_{0} \varphi(\bar{x})$ called the Fréchet subdifferential of $\varphi$ at $\bar{x}$. If $\varphi$ is Fréchet differentiable at $\bar{x}$, then $\widehat{\partial} \varphi(\bar{x})$ reduces to the classical Fréchet derivative. In general the set $\widehat{\partial}_{\varepsilon} \varphi(\bar{x})$ is convex for any $\varepsilon \geq 0$ while it may often be empty for nonconvex functions as, e.g., in the case of $\varphi(x)=-|x|$ at $\bar{x}=0 \in \mathbb{R}$. Observe furthermore that the subdifferential construction $(2.8)$, including $\widehat{\partial} \varphi(\cdot)$, does not satisfy pointwise calculus rules (for sums, compositions, etc.) required in various applications. This is dramatically improved for the sequential limiting construction

$$
\partial \varphi(\bar{x}):=w^{*}-\operatorname{Limsup}_{\substack{x \rightarrow \bar{x} \\ \varepsilon \downarrow 0}} \widehat{\partial}_{\varepsilon} \varphi(x),
$$


known as the basic/limiting/Mordukhovich subdifferential of $\varphi$ at $\bar{x}$, where $x \stackrel{\varphi}{\rightarrow} \bar{x}$ stands for $x \rightarrow \bar{x}$ with $\varphi(x) \rightarrow \varphi(\bar{x})$. We can equivalently put $\varepsilon=0$ in (2.9) if $\varphi$ is l.s.c. around $\bar{x}$ and the space $X$ is Asplund, i.e., each separable subspace of it has a separable dual. The latter subclass of Banach spaces is sufficiently large including all reflexive spaces and all Banach spaces with a separable dual. Note that the basic subdifferential (2.9) reduces to the classical derivative for smooth functions $\varphi$ and to the subdifferential of convex analysis when $\varphi$ is convex, while the set (2.9) may often be nonconvex for simple nonconvex functions; e.g., $\partial \varphi(0)=\{-1,1\}$ for $\varphi(x)=-|x|$. Recall also that $\partial \varphi(\bar{x}) \neq \emptyset$ if $\varphi$ is locally Lipschitzian around $\bar{x}$ and the space $X$ is Asplund; see [14, Corollary 2.25].

It is clear from (2.9) that we always have the inclusion

$$
\widehat{\partial} \varphi(\bar{x}) \subset \partial \varphi(\bar{x}), \quad \bar{x} \in \operatorname{dom} \varphi .
$$

A function $\varphi: X \rightarrow \overline{\mathbb{R}}$ is called to be lower regular at $\bar{x}$ if (2.10) holds as equality. Besides smooth functions, convex functions and the like, the latter property is satisfied for various classes of "nice" functions important in applications. Furthermore, there is a well-developed calculus ensuring the preservation of lower regularity under a variety of operations; see [14] for more details. We can easily deduce from the previous discussions that $\widehat{\partial} \varphi(\bar{x}) \neq \emptyset$ if $\varphi$ is locally Lipschitzian around $\bar{x}$ and lower regular at this point provided that either $X$ is Asplund, or $\varphi$ is convex. It is important in the framework of this paper, where the minimal time function (1.2) is Lipschitz continuous on $X$ while its Fréchet subdifferentiability $\widehat{\partial} T(\bar{x}) \neq \emptyset$ is used in the major results established below.

Given further a function $\varphi: X \rightarrow \mathbb{R}$ on a Banach space $X$ locally Lipschitzian around $\bar{x}$, recall the Clarke generalized gradient of $\varphi$ at $\bar{x}$ defined by

$$
\bar{\partial} \varphi(\bar{x}):=\left\{x^{*} \in X^{*} \mid\left\langle x^{*}, v\right\rangle \leq \varphi^{\circ}(\bar{x} ; v) \text { for all } v \in X\right\},
$$

where the generalized directional derivative of $\varphi$ at $\bar{x}$ in direction $v \in X$ is

$$
\varphi^{\circ}(\bar{x} ; v):=\limsup _{\substack{x \rightarrow \bar{x} \\ t \downarrow 0}} \frac{\varphi(x+t v)-\varphi(x)}{t} .
$$

The reader is referred to [5] for more details on these constructions. If $X$ is Asplund, we have the relationship [14, Theorem 3.57]

$$
\bar{\partial} \varphi(\bar{x})=\operatorname{cl}^{*} \operatorname{co} \partial \varphi(\bar{x})
$$

between (2.11) and our basic subdifferential (2.9), where $\mathrm{cl}^{*}$ co stands for the convex closure of the set in the weak* topology of $X^{*}$. It follows from (2.12) and the aforementioned nonemptiness of the basic subdifferential that $\bar{\partial} \varphi(\bar{x}) \neq \emptyset$ for locally Lipschitzian functions on Asplund spaces.

Finally in this section, define the normal cones

$$
\widehat{N}(\bar{x} ; \Omega):=\widehat{\partial} \delta_{\Omega}(\bar{x}) \text { and } N(\bar{x} ; \Omega):=\partial \delta_{\Omega}(\bar{x})
$$


to a set $\Omega \subset X$ at $\bar{x} \in \Omega$ generated by the corresponding subdifferentials (2.8) and (2.9) of the indicator function of $\Omega$. Note that for $X=\mathbb{R}^{n}$ the limiting normal cone in (2.13) can be equivalently described via the metric projection (1.6) by

$$
N(\bar{x} ; \Omega)=\operatorname{Limsup}_{x \rightarrow \bar{x}}\left[\operatorname{cone}\left(x-\Pi_{\Omega}(x)\right)\right],
$$

where the symbol "cone" stands for the conic hull of the set. We say that $\Omega$ is normally regular at $\bar{x} \in \Omega$ if $\widehat{N}(\bar{x} ; \Omega)=N(\bar{x} ; \Omega)$. This property holds for convex and "nice" nonconvex sets; it satisfies rich calculus ensuring its preservation under various operations.

\section{General Representation Formula}

Our first result is the following unconditional inclusion, which is definitely of its own interest being at the same time crucial for the subsequent applications in this paper.

Theorem 3.1 (approximate projection via minimal time function and duality mapping in Banach spaces). For each $\bar{x} \notin C$ we have the inclusion

$$
w-\operatorname{Limsup} \Pi_{\varepsilon \downarrow 0}^{F}(\bar{x}, \varepsilon) \subset \bigcap_{x^{*} \in \widehat{\partial} T(\bar{x})}\left(\bar{x}+T(\bar{x}) \mathfrak{J}_{F}\left(-x^{*}\right)\right) .
$$

Proof. In the case of $\widehat{\partial} T(\bar{x})=\emptyset$ inclusion (3.1) is trivial, since the right-hand side of (3.1) is the whole space $X$ by the standard convention. Assuming that $\widehat{\partial} T(\bar{x}) \neq \emptyset$, we have $\widehat{\partial} T(\bar{x}) \subset-\operatorname{bd} F^{0}$ by [12, Theorem 4.2], which is a Banach space extension of the Hilbert space result of [9, Theorem 3.1]. This implies by (2.2) that

$$
\mathfrak{J}_{F}\left(-x^{*}\right)=\left\{x \in F \mid\left\langle-x^{*}, x\right\rangle=1\right\} \text { for }-x^{*} \in \widehat{\partial} T(\bar{x}) .
$$

Select now an arbitrary element $y \in w-\operatorname{Limsup}_{\varepsilon \downarrow 0} \Pi_{C}^{F}(\bar{x}, \varepsilon)$ and find by (2.6) a sequence $\left\{y_{k}\right\}$ with $y_{k} \in \Pi_{C}^{F}\left(\bar{x}, \varepsilon_{k}\right)$ and $\varepsilon_{k} \downarrow 0$ such that $y_{k} \stackrel{w}{\rightarrow} y$ as $k \rightarrow \infty$. It follows from the definition that $T(\bar{x}) \leq \rho_{F}\left(y_{k}-\bar{x}\right) \leq T(\bar{x})+t_{k}^{2}$ with $t_{k}:=\varepsilon_{k}^{1 / 2}>0$. Taking an arbitrary Fréchet subgradient $x^{*} \in \widehat{\partial} T(\bar{x})$, we get from (2.8) as $\varepsilon=0$ that

$$
\liminf _{x \rightarrow \bar{x}} \frac{T(x)-T(\bar{x})-\left\langle x^{*}, x-\bar{x}\right\rangle}{\|x-\bar{x}\|} \geq 0
$$

Define further a sequence $\left\{x_{k}\right\} \subset X$ by

$$
x_{k}:=\bar{x}+t_{k}\left(y_{k}-\bar{x}\right), \quad k \in \mathbb{N},
$$

and observe that $x_{k} \rightarrow \bar{x}$ as $k \rightarrow \infty$, since $\left\{y_{k}\right\}$ is bounded in $X$. Furthermore, $x_{k} \neq \bar{x}$ for all $k \in \mathbb{N}$ by construction. It thus follows from (3.3) that there is a sequence $\left\{\alpha_{k}\right\} \subset \mathbb{R}$ with $\lim _{k \rightarrow \infty} \alpha_{k} \geq 0$ for which we have the inequality

$$
\frac{T\left(x_{k}\right)-T(\bar{x})}{t_{k}} \geq\left\langle x^{*}, y_{k}-\bar{x}\right\rangle+\alpha_{k}, \quad k \in \mathbb{N} .
$$


The latter implies by the definitions of the minimal time and Minkowski functions and by the constructions of the sequences involved that

$$
\left\langle x^{*}, y_{k}-\bar{x}\right\rangle+\alpha_{k} \leq \frac{\rho_{F}\left(y_{k}-x_{k}\right)-\rho_{F}\left(y_{k}-\bar{x}\right)+t_{k}^{2}}{t_{k}}=-\rho_{F}\left(y_{k}-\bar{x}\right)+t_{k}, \quad k \in \mathbb{N} .
$$

By passing to the "limsup" as $k \rightarrow \infty$ above, we arrive at

$$
\liminf _{k \rightarrow \infty}\left\langle x^{*}, \bar{x}-y_{k}\right\rangle \geq \lim _{k \rightarrow \infty} \rho_{F}\left(y_{k}-\bar{x}\right)=T(\bar{x}) .
$$

On the other hand, by the choice of $x^{*}$ we have the relationships

$$
\left\langle x^{*}, \bar{x}-y_{k}\right\rangle=\left\langle-x^{*}, y_{k}-\bar{x}\right\rangle \leq \sigma_{F^{0}}\left(y_{k}-\bar{x}\right)=\rho_{F}\left(y_{k}-\bar{x}\right),
$$

which imply, by passing to the "lim sup" as $k \rightarrow \infty$, that

$$
\limsup _{k \rightarrow \infty}\left\langle x^{*}, \bar{x}-y_{k}\right\rangle \leq \lim _{k \rightarrow \infty} \rho_{F}\left(y_{k}-\bar{x}\right)=T(\bar{x})
$$

Comparing (3.5) with (3.4) allows us to conclude that the limit $\lim _{k \rightarrow \infty}\left\langle x^{*}, \bar{x}-y_{k}\right\rangle$ exists and equals $T(\bar{x})$. This implies, since the sequence $\left\{y_{k}\right\}$ weakly converges in $X$ to the element $y$ fixed above, that

$$
\left\langle x^{*}, \bar{x}-y_{k}\right\rangle \rightarrow\left\langle x^{*}, \bar{x}-y\right\rangle \text { as } k \rightarrow \infty,
$$

and thus $\left\langle x^{*}, \bar{x}-y\right\rangle=T(\bar{x})$. Furthermore, we have

$$
\lim _{k \rightarrow \infty} \rho_{F}\left(y_{k}-\bar{x}\right)=T(\bar{x})=\left\langle x^{*}, \bar{x}-y\right\rangle \leq \sigma_{F^{0}}(y-\bar{x})=\rho_{F}(y-\bar{x}) .
$$

By its convexity, the function $\rho_{F}(\cdot)$ is weakly lower semicontinuous on $X$. Hence

$$
\liminf _{k \rightarrow \infty} \rho_{F}\left(y_{k}-\bar{x}\right) \geq \rho_{F}(y-\bar{x}) .
$$

Combining the relationships in (3.6) and (3.7) gives us

$$
\lim _{k \rightarrow \infty} \rho_{F}\left(y_{k}-\bar{x}\right)=\rho_{F}(y-\bar{x})=T(\bar{x}),
$$

which in turn implies that

$$
\left\langle x^{*}, \frac{\bar{x}-y}{\rho_{F}(y-\bar{x})}\right\rangle=1 .
$$

Since $-x^{*} \in \operatorname{bd} F^{0}$ by the above, we get the inclusion

$$
\frac{y-\bar{x}}{\rho_{F}(y-\bar{x})} \in \mathfrak{J}_{F}\left(-x^{*}\right), \text { i.e., } y \in \bar{x}+T(\bar{x}) \mathfrak{J}_{F}\left(-x^{*}\right),
$$

which concludes the proof of the theorem by taking into account that the Fréchet subgradient $-x^{*} \in \widehat{\partial} T(\bar{x})$ was chosen arbitrarily.

As a consequence of Theorem 3.1, we obtain a precise representation of the (possibly empty) minimal time projection (1.4) in arbitrary Banach spaces under our standing assumptions made on the dynamics and target. 
Corollary 3.2 (precise representation of minimal time projection in Banach spaces). For each $\bar{x} \notin C$ with $\widehat{\partial} T(\bar{x}) \neq \emptyset$ the following holds: if $x^{*} \in \widehat{\partial} T(\bar{x})$ then

$$
\Pi_{C}^{F}(\bar{x})=\left(\bar{x}+T(\bar{x}) \mathfrak{J}_{F}\left(-x^{*}\right)\right) \cap C .
$$

Proof. It follows from Theorem 3.1 that for any fixed $\bar{x} \notin C$ and $x^{*} \in \widehat{\partial} T(\bar{x})$ we have

$$
\Pi_{C}^{F}(\bar{x}) \subset w-\operatorname{Limsup}_{\varepsilon \downarrow 0} \Pi_{C}^{F}(\bar{x}, \varepsilon) \subset \bar{x}+T(\bar{x}) \mathfrak{J}_{F}\left(-x^{*}\right) .
$$

This implies, by taking into account the obvious one $\Pi_{C}^{F}(\bar{x}) \subset C$, that the inclusion " $\subset$ " holds in (3.8). To justify the opposite inclusion " $\supset$ " in (3.8), observe that for any

$$
y \in\left(x+T(\bar{x}) \mathfrak{J}_{F}\left(-x^{*}\right)\right) \cap C \subset(\bar{x}+T(\bar{x}) \mathrm{bd} F) \cap C
$$

we get by the constructions of (1.2) and (2.2) with $x^{*} \in \widehat{\partial} T(\bar{x}) \subset-\operatorname{bd} F^{\circ}$ that

$$
y \in C \text { and } \rho_{F}(y-\bar{x})=T(\bar{x}),
$$

which gives $y \in \Pi_{C}^{F}(\bar{x})$ and thus completes the proof of the corollary.

\section{Minimal Time Projection via Dynamics Rotundity}

In this section we derive and illustrate sufficient conditions ensuring the existence and uniqueness of the minimal time projection (1.4) in reflexive Banach spaces under certain rotundity properties of the dynamics $F$. We say that $F$ is strongly rotund with respect to $x^{*} \in \mathrm{bd} F^{0}$ if there is a (unique) point $\bar{x} \in \mathrm{bd} F$ such that the rotundity modulus

$$
\mathfrak{R}_{r}\left(\bar{x}, x^{*}\right):=\inf \left\{\left\langle x^{*}, \bar{x}-x\right\rangle: x \in F,\|x-\bar{x}\| \geq r\right\}
$$

is positive for each $r>0$. Some characterizations and verifiable sufficient conditions ensuring this property in a Hilbert space setting are given in [10] and can be easily extended to the case of an arbitrary Banach space; see also [18, Proposition 5.11]. Note that our rotundity condition is equivalent to the Fréchet differentiability of the support function $\sigma_{F}(\cdot)$ at $x^{*}$, and in this case $\bar{x}=\nabla \sigma_{F}\left(x^{*}\right)$. In turn, the condition $\mathfrak{R}_{r}\left(\bar{x}, x^{*}\right)>0$ is equivalent to saying that $\bar{x}$ is a strongly exposed point of the set $F$ (see [18]) in the sense that the hyperplane

$$
H\left(x^{*}\right):=\left\{x \in X:\left\langle x^{*}, x\right\rangle=\sigma_{F}\left(x^{*}\right)\right\}
$$

touches $F$ at the point $\bar{x}$ only (i.e., $\mathfrak{J}_{F}\left(x^{*}\right)=\{\bar{x}\}$ ) and that each sequence $\left\{x_{k}\right\} \subset F$ with $\left\langle x^{*}, x_{k}-\bar{x}\right\rangle \rightarrow 0$ strongly converges to $\bar{x}$ as $k \rightarrow \infty$. In fact, the latter property will be used in the proof of Theorem 4.1 below. However, it is preferable to formulate the main hypothesis in terms of the Fréchet differentiability of $\sigma_{F}$, since in this case we do not refer to any point $\bar{x} \in \mathrm{bd} F$. Observe furthermore that the following geometrical property is sufficient for the strong rotundity (see [10, Section 3]): taking the (unique) point $\bar{x} \in \operatorname{bd} F$ at which $x^{*}$ is normal to $F$, we have

$$
\sup _{\|x-\bar{x}\| \geq r} \rho_{F}(x+\bar{x})<2 \text { for each } r>0 .
$$


Theorem 4.1 (existence and uniqueness of minimal time projection for rotund dynamics). Let $X$ be reflexive, and let $\bar{x} \notin C$ with $\widehat{\partial} T(\bar{x}) \neq \emptyset$. Assume in addition that for some $x^{*} \in \widehat{\partial} T(\bar{x})$ the support function $\sigma_{F}(\cdot)$ of the dynamics is Fréchet differentiable at $-x^{*}$. Then the set $\Pi_{C}^{F}(\bar{x})$ is a singleton.

Proof. Since the approximate projection (2.5) is a nonempty, closed, and bounded subset of $X$ for any $\varepsilon>0$, we deduce from the reflexivity of $X$ that the weak sequential outer limit $w$-Lim $\sup \Pi_{C}^{F}(\bar{x}, \varepsilon)$ is a nonempty subset of $X$. Pick any element $y$ from the $\varepsilon \downarrow 0$

latter set that surely belongs to $\overline{\mathrm{co}} C$, the convex closure of $C$. We have by Theorem 3.1 that $y \in \bar{x}+T(\bar{x}) \mathfrak{J}_{F}\left(-x^{*}\right)$. Furthermore, by the Fréchet differentiability of $\sigma_{F}(\cdot)$ (or, equivalently, of $\left.\rho_{F^{0}}(\cdot)\right)$ at $-x^{*}$, it follows from [18, Proposition 5.11] that $\mathfrak{J}_{F}\left(-x^{*}\right)$ is a singleton $\{v\}$, which is a strongly exposed point of $F$. By the above choice of $y$ we conclude that $y$ is a weak limit of a minimizing sequence $\left\{y_{k}\right\} \subset C$ with $\rho_{F}\left(y_{k}-\bar{x}\right) \rightarrow T(\bar{x})$ as $k \rightarrow \infty$. Then we have the weak convergence

$$
\frac{y_{k}-\bar{x}}{\rho_{F}\left(y_{k}-\bar{x}\right)} \stackrel{w}{\longrightarrow} \frac{y-\bar{x}}{T(\bar{x})}=v \text { as } k \rightarrow \infty .
$$

Since $v$ is a strongly exposed point of $F$, the convergence in (4.2) is indeed strong in $X$, and thus $y_{k} \rightarrow y$ as $k \rightarrow \infty$. This gives $y \in C$, which yields that $y \in \Pi_{C}^{F}(\bar{x}) \neq \emptyset$ by Corollary 3.2. Observe finally that the above arguments with the usage of Corollary 3.2 ensure in fact that the minimal time projection set $\Pi_{C}^{F}(\bar{x})$ is a singleton.

Remark 4.2 (on Fréchet subdifferentiability and lower regularity). Since any reflexive space is Asplund and since the minimal time function (1.2) is Lipschitz continuous, $T(\cdot)$ is Fréchet subdifferentiable $(\widehat{\partial} T(\bar{x}) \neq \emptyset)$ at a point $\bar{x}$ if, in particular, it is lower regular at this point. Indeed, this follows from the definition of lower regularity in Section 2 and the fact that the basic subdifferential (2.9) is nonempty for any locally Lipschitzian function on an Asplund space; see [14, Corollary 2.25].

Let us show next that the Fréchet differentiability assumption on $\sigma_{F}(\cdot)$ in Theorem 4.1 cannot be removed, for a separable infinite-dimensional Hilbert space $X$, in order to ensure the existence of the minimal time projection (1.4). Furthermore, the generalized projection may exist without this assumption while not being unique.

Example 4.3 (non-existence and non-uniqueness of generalized projection for dynamics with nonsmooth support function). In a separable Hilbert space $X$ there are dynamics sets $F \subset X$ and target sets $C \subset X$ satisfying our standing assumptions and such that $T(\cdot)$ is Fréchet differentiable at $0, \sigma_{F}(\cdot)$ is not Fréchet differentiable at $-\nabla T(0)$ while $\Pi_{C}^{F}(0)=\emptyset$. Furthermore, there are $F$ and $C$ with the above properties for which $\Pi_{C}^{F}(0) \neq \emptyset$ while the latter projection set is not a singleton.

Proof. Let $X$ be a separable infinite-dimensional Hilbert space with an orthonormal base $\left\{e_{n}\right\} \subset X$. Define the dynamics and target sets by, respectively,

$$
F:=\left\{x \in X \mid\|x\| \leq 1,\left\langle x, e_{1}\right\rangle \leq \frac{1}{2}\right\}
$$




$$
C:=\left\{\left(\frac{1}{2}+\frac{1}{n}\right)\left(\frac{e_{n}}{2}+e_{1}\right) \mid n \geq 2\right\} .
$$

It is easy to check that the sets $F$ and $C$ satisfy our standing assumptions formulated in Section 1. Observe that $T(x)=1-2\left\langle x, e_{1}\right\rangle$ in a neighborhood of the origin, and so the minimal time function is Fréchet differentiable therein. We have $T(0)=1$ and $\nabla T(0)=-2 e_{1}$ while $\rho_{F}(y)>1$ for all $y \in C$. The latter means that the minimum of $\rho_{F}(\cdot)$ is not attained in $C$, and thus $\Pi_{C}^{F}(0)=\emptyset$. Observe furthermore that

$$
\mathfrak{J}_{F}(-\nabla T(0))=\left\{x \in X \mid\|x\| \leq 1,\left\langle x, e_{1}\right\rangle=\frac{1}{2}\right\}
$$

which contains, in particular, the points $\frac{1}{2} e_{1}+\lambda e_{n}$ with $\lambda^{2} \leq 3 / 4, n=2,3, \ldots$. Thus the support function $\sigma_{F}(\cdot)$ is not Fréchet differentiable at $-\nabla T(0)$.

By keeping the same dynamics (4.3) while changing the target (4.4) by

$$
\widetilde{C}:=\left\{\left(\frac{1}{2}+\frac{1}{n}\right) \frac{e_{n}}{2} \mid n \geq 2\right\},
$$

we easily check that the minimal time projection $\Pi_{C}^{F}(0)$ is nonempty, but it is not a singleton. In this case the minimal time function $T(\cdot)$ remains the same.

\section{Minimal Time Projection via Target Set}

In this section we derive new verifiable conditions for the existence and uniqueness of the minimal time projection (1.4) that, in contrast to the "dynamics" ones in Section 4, exploit similar properties of the target set $C$. Some of the results obtained are necessary and sufficient for the generalized projection set $\Pi_{C}^{F}(\bar{x})$ to be a singleton. As in Section 4, we essentially use here the underlying projection formula established above in Corollary 3.2 applying it to a modified projection operator.

First we use Corollary 3.2 to interpret the minimal time function (1.2) and the minimal time projection (1.4) in a somewhat different way. Assuming that $\widehat{\partial} T(\bar{x}) \neq \emptyset$ for some $\bar{x} \in X$ and picking a Fréchet subgradient $x^{*} \in \widehat{\partial} T(\bar{x})$, consider the modified minimal time problem

$$
\text { minimize } t>0 \text { subject to }\left(x+t \mathfrak{J}_{F}\left(-x^{*}\right)\right) \cap C \neq \emptyset, \quad x \in X,
$$

for which the dynamics $F$ in (1.1) is replaced by the face of $F$ corresponding to the support functional $-x^{*}$. Denote the modified minimal time function in (5.1) by

$$
\widetilde{T}_{x^{*}}(x)=\inf _{y \in C} \rho_{\mathfrak{J}_{F}\left(-x^{*}\right)}(y-x)
$$

and the corresponding modified minimal time projection operator by

$$
\widetilde{\Pi}_{C}^{F}\left(x, x^{*}\right):=\left\{y \in C \mid \rho_{\mathfrak{J}_{F}\left(-x^{*}\right)}(y-x)=\widetilde{T}_{x^{*}}(x)\right\} .
$$

The next proposition provides conditions for the nonemptiness of the original minimal time projection (1.4) in terms of the modified problem (5.1). 
Proposition 5.1 (existence of minimal time projection via modified problem). For any fixed $\bar{x} \in X$ such that $\widehat{\partial} T(\bar{x}) \neq \emptyset$ and $x^{*} \in \widehat{\partial} T(\bar{x})$ we have that $\Pi_{C}^{F}(\bar{x}) \neq \emptyset$ if and only if $T(\bar{x})=\widetilde{T}_{x^{*}}(\bar{x})$ and $\widetilde{\Pi}_{C}^{F}\left(\bar{x}, x^{*}\right) \neq \emptyset$. Furthermore, $\widetilde{\Pi}_{C}^{F}\left(\bar{x}, x^{*}\right)=\Pi_{C}^{F}(\bar{x})$ whenever these conditions hold.

Proof. With no loss of generality, suppose that $\bar{x} \notin C$. Assuming that $\Pi_{C}^{F}(\bar{x}) \neq \emptyset$ and taking the selected subgradient $x^{*} \in \widehat{\partial} T(\bar{x})$ give us by Corollary 3.2 that

$$
\left(\bar{x}+T(\bar{x}) \mathfrak{J}_{F}\left(-x^{*}\right)\right) \cap C \neq \emptyset \text {, and hence } \widetilde{T}_{x^{*}}(\bar{x}) \leq T(\bar{x}) .
$$

On the other hand, we have the relationships

$$
\mathfrak{J}_{F}\left(-x^{*}\right) \subset F, \text { and thus } \rho_{\mathfrak{J}_{F}\left(-x^{*}\right)}(y-\bar{x}) \geq \rho_{F}(y-\bar{x}) \text { for all } y \in C \text {. }
$$

Taking the infimum in (5.4) over $y \in C$ gives us $\widetilde{T}_{x^{*}}(\bar{x}) \geq T(\bar{x})$. Thus we get

$$
\widetilde{T}_{x^{*}}(\bar{x})=T(\bar{x}) \text { and } \widetilde{\Pi}_{C}^{F}\left(\bar{x}, x^{*}\right)=C \cap\left(\bar{x}+\widetilde{T}_{x^{*}}(\bar{x}) \mathfrak{J}_{F}\left(-x^{*}\right)\right)=\Pi_{C}^{F}(\bar{x}) \neq \emptyset,
$$

which proves the "only if" part of the proposition.

To justify the "if" part, suppose that $T(\bar{x})=\widetilde{T}_{x^{*}}(\bar{x})$ and $\widetilde{\Pi}_{C}^{F}\left(\bar{x}, x^{*}\right) \neq \emptyset$. Then we easily conclude by Corollary 3.2 that

$$
\begin{aligned}
\Pi_{C}^{F}(\bar{x}) & =\left(\bar{x}+T(\bar{x}) \mathfrak{J}_{F}\left(-x^{*}\right)\right) \cap C \\
& =\left(\bar{x}+\widetilde{T}_{x^{*}}(\bar{x}) \mathfrak{J}_{F}\left(-x^{*}\right)\right) \cap C=\widetilde{\Pi}_{C}^{F}\left(\bar{x}, x^{*}\right) \neq \emptyset,
\end{aligned}
$$

which thus completes the proof of the proposition.

To proceed with deriving sufficient conditions for well-posedness of the minimal time problem (1.1) in terms of the target set $C$, we need the following representation (Proposition 5.2) of the modified projection (5.3) via the support function of some subset of the target. Given $x^{*} \in \widehat{\partial} T(\bar{x})$, denote by $K_{x^{*}}$ the convex and closed cone generated by the duality mapping value $\mathfrak{J}_{F}\left(-x^{*}\right)$. Consider further the set

$$
C\left(\bar{x}, x^{*}\right):=C \cap\left(\bar{x}+K_{x^{*}}\right),
$$

which is nonempty if and only if $\widetilde{T}_{x^{*}}(\bar{x})<\infty$ for the modified minimal time function (5.2). In particular, it follows from Proposition 5.1 that $C\left(\bar{x}, x^{*}\right) \neq \emptyset$ if $\Pi_{C}^{F}(\bar{x}) \neq \emptyset$.

Proposition 5.2 (representation of modified minimal time projection in Banach spaces). Given $\bar{x} \notin C$ and $x^{*} \in \widehat{\partial} T(\bar{x})$, we have the representation

$$
\widetilde{\Pi}_{C}^{F}\left(\bar{x}, x^{*}\right)=\left\{y \in C\left(\bar{x}, x^{*}\right) \mid \sigma_{C\left(\bar{x}, x^{*}\right)}\left(x^{*}\right)=\left\langle x^{*}, y\right\rangle\right\}
$$

of the modified projection (5.3) via the support function of (5.5). 
Proof. If $y$ belongs to the right-hand side of (5.6), then

$$
\left\langle x^{*}, y\right\rangle \geq\left\langle x^{*}, v\right\rangle \text { for all } v \in C \text { with } v-\bar{x} \in K_{x^{*}} .
$$

Moreover, $y \in C\left(\bar{x}, x^{*}\right)$, i.e., $y \in C$ and there exists $\lambda>0$ with $y \in \bar{x}+\lambda \mathfrak{J}_{F}\left(-x^{*}\right)$.

Let us show that $\lambda=\widetilde{T}_{x^{*}}(\bar{x})$. Indeed, we have $\left\langle-x^{*}, y-\bar{x}\right\rangle=\lambda$, and thus inequality (5.7) can be rewritten as follows:

$$
\lambda \leq t \text { for all } t>0 \text { such that } C \cap\left(\bar{x}+t \mathfrak{J}_{F}\left(-x^{*}\right)\right) \neq \emptyset .
$$

This implies that $\lambda \leq \widetilde{T}_{x^{*}}(\bar{x})$, since $\widetilde{T}_{x^{*}}(\bar{x})$ is the infimum of all such $t>0$ by (5.1) and (5.2). On the other hand, we have

$$
y \in\left(\bar{x}+\lambda \mathfrak{J}_{F}\left(-x^{*}\right)\right) \cap C \neq \emptyset,
$$

which yields $\widetilde{T}_{x^{*}}(\bar{x}) \leq \lambda$. Thus $\lambda=\widetilde{T}_{x^{*}}(\bar{x})<\infty$, and furthermore

$$
\rho_{\mathfrak{J}_{F}\left(-x^{*}\right)}(y-\bar{x}) \leq \lambda=\widetilde{T}_{x^{*}}(\bar{x}) \text {, i.e., } y \in \widetilde{\Pi}_{C}^{F}\left(\bar{x}, x^{*}\right) .
$$

This gives $y \in \widetilde{\Pi}_{C}^{F}\left(\bar{x}, x^{*}\right)$ and justifies the inclusion " $\supset$ " in (5.6).

To prove the inclusion " $\subset$ " in (5.6), pick any $y \in \widetilde{\Pi}_{C}^{F}\left(\bar{x}, x^{*}\right)$ and get by (5.3) that

$$
\rho_{\mathfrak{J}_{F}\left(-x^{*}\right)}(y-\bar{x}) \leq \rho_{\mathfrak{J}_{F}\left(-x^{*}\right)}(v-\bar{x}) \text { for all } v \in C .
$$

Define further $\lambda:=\widetilde{T}_{x^{*}}(\bar{x})=\rho_{\mathfrak{J}_{F}\left(-x^{*}\right)}(y-\bar{x})$ and observe that $y \in \bar{x}+\lambda \mathfrak{J}_{F}\left(-x^{*}\right)$. On the other hand, take $t>0$ to be sufficiently small to have $C \cap\left(\bar{x}+t \mathfrak{J}_{F}\left(-x^{*}\right)\right) \neq \emptyset$. In this case there is $v \in C$ with $t=\left\langle-x^{*}, v-\bar{x}\right\rangle$, and we get $\lambda \leq t$. Taking now into account that $\lambda=\left\langle-x^{*}, y-\bar{x}\right\rangle$ by construction, we arrive at

$$
\left\langle x^{*}, y-\bar{x}\right\rangle \geq\left\langle x^{*}, v-\bar{x}\right\rangle \text { for each } v \in C \text { with } v-\bar{x} \in t \mathfrak{J}_{F}\left(-x^{*}\right), t>0 .
$$

It gives $\left\langle x^{*}, y\right\rangle \geq\left\langle x^{*}, v\right\rangle$ whenever $v \in C \cap\left(\bar{x}+K_{x^{*}}\right)$, which justifies the inclusion " $\subset$ " in (5.6) and completes the proof of the proposition.

Next we use the above propositions to derive necessary and sufficient conditions for the existence and uniqueness of the minimal time projection (1.4) at the point in question involving the modified value function (5.2) and appropriate subsets of the target set $C$. Note the results obtained in the following theorem are new for the uniqueness part even in the cases when the existence is already known as, e.g., in finite dimensions as well as for convex target sets in reflexive spaces.

Theorem 5.3 (characterization of well-posedness of minimal time projection via subsets of target). Let $X$ be a reflexive Banach space, and let $\bar{x} \notin C$ be such that $\widehat{\partial} T(\bar{x}) \neq \emptyset$. Then the minimal time projection set $\Pi_{C}^{F}(\bar{x})$ is a singleton if and only if there exists $x^{*} \in \widehat{\partial} T(\bar{x})$ such that $\widetilde{T}_{x^{*}}(\bar{x})=T(\bar{x})$ and the support function $\sigma_{C\left(\bar{x}, x^{*}\right)}(\cdot)$ of the set $C\left(\bar{x}, x^{*}\right)$ from (5.5) is Gâteaux differentiable at $x^{*}$. In this case we have

$$
\Pi_{C}^{F}(\bar{x})=\left\{\nabla \sigma_{C\left(\bar{x}, x^{*}\right)}\left(x^{*}\right)\right\} .
$$


Proof. By Proposition 5.1 we have that the set $\Pi_{C}^{F}(\bar{x})$ is nonempty if and only if $\widetilde{\Pi}_{C}^{F}\left(\bar{x}, x^{*}\right) \neq \emptyset$ and $\widetilde{T}_{x^{*}}(\bar{x})=T(\bar{x})$ for some $x^{*} \in \widehat{\partial} T(\bar{x})$. It holds furthermore that $\widetilde{\Pi}_{C}^{F}\left(\bar{x}, x^{*}\right)=\Pi_{C}^{F}(\bar{x})$, and thus the sets $\Pi_{C}^{F}(\bar{x})$ and $\widetilde{\Pi}_{C}^{F}\left(\bar{x}, x^{*}\right)$ are singletons simultaneously. Employing Proposition 5.2, we get in this case that the right-hand side of (5.6) reduces to $\{\bar{y}\}$ for some $\bar{y} \in C\left(\bar{x}, x^{*}\right)$. On the other hand, the subdifferential of the (always convex) support function $\sigma_{C\left(\bar{x}, x^{*}\right)}(\cdot)$ at $x^{*}$ admits the representation

$$
\partial \sigma_{C\left(\bar{x}, x^{*}\right)}\left(x^{*}\right)=\partial \sigma_{\overline{\mathrm{co}} C\left(\bar{x}, x^{*}\right)}\left(x^{*}\right)=\left\{v \in \overline{\mathrm{co}} C\left(\bar{x}, x^{*}\right) \mid \sigma_{C\left(\bar{x}, x^{*}\right)}\left(x^{*}\right)=\left\langle x^{*}, v\right\rangle\right\} .
$$

It follows from (5.9) that the set $\widetilde{C}:=\partial \sigma_{C\left(\bar{x}, x^{*}\right)}\left(x^{*}\right)$ is an exposed face of the convex closed set $\overline{\mathrm{co}} C\left(\bar{x}, x^{*}\right)$.

Since $\left\langle x^{*}, v\right\rangle=\left\langle x^{*}, \bar{y}\right\rangle$ for each $v \in \tilde{C}$ and $\bar{y} \in \bar{x}+T(\bar{x}) \mathfrak{J}_{F}\left(-x^{*}\right)$ by Corollary 3.2, we have that $\tilde{C} \subset \bar{x}+[0, T(\bar{x})] \mathfrak{J}_{F}\left(-x^{*}\right)$, which ensures the boundedness of the set $\widetilde{C}$ in $X$. The reflexivity of the space $X$ and the convexity and closedness of $\widetilde{C}$ allow us to conclude that the latter set is weakly compact in $X$. By the classical Kreln-Mil'man theorem there is an extreme point $\bar{v}$ of $\widetilde{C}$ that is, by transitivity, an extreme point of the set $\overline{c o} C\left(\bar{x}, x^{*}\right)$ as well. Thus we have $\bar{v} \in C\left(\bar{x}, x^{*}\right)$, and the equality $\sigma_{C\left(\bar{x}, x^{*}\right)}\left(x^{*}\right)=\left\langle x^{*}, \bar{v}\right\rangle$ holds. The latter means that $\bar{v}$ belongs to the set on the right-hand side of (5.6), which is the singleton $\{\bar{y}\}$ in the notation above. This justifies that the subgradient set $\widetilde{C}=\partial \sigma_{C\left(\bar{x}, x^{*}\right)}\left(x^{*}\right)$ is a singleton, and thus - by the classical result of convex analysis - the support function $\sigma_{C\left(\bar{x}, x^{*}\right)}(\cdot)$ is Gâteaux differentiable at $x^{*}$ with

$$
\Pi_{C}^{F}(\bar{x})=\{\bar{y}\}=\{\bar{v}\}=\left\{\nabla \sigma_{C\left(\bar{x}, x^{*}\right)}\left(x^{*}\right)\right\} .
$$

This proves the "only if" part of the theorem with the projection representation (5.8).

To justify the "if" part, suppose that the support function $\sigma_{C\left(\bar{x}, x^{*}\right)}(\cdot)$ is Gâteaux differentiable at $x^{*}$, which gives the relationships

$$
\partial \sigma_{C\left(\bar{x}, x^{*}\right)}\left(x^{*}\right)=\left\{\nabla \sigma_{C\left(\bar{x}, x^{*}\right)}\left(x^{*}\right)\right\}=\{\bar{y}\} .
$$

Thus $\bar{y}$ is an exposed point of the convex weakly compact set $\overline{\operatorname{co}} C\left(\bar{x}, x^{*}\right)$. This gives $\bar{y} \in$ $C\left(\bar{x}, x^{*}\right)$ and yields, by using formula (5.6) from Proposition 5.2, that $\widetilde{\Pi}_{C}^{F}\left(\bar{x}, x^{*}\right)=\{\bar{y}\}$. Taking finally into account that $\widetilde{T}_{x^{*}}(\bar{x})=T(\bar{x})$ as assumed, we arrive at $\Pi_{C}^{F}(\bar{x})=\{\bar{y}\}$ and complete the proof of the theorem.

Observe further that, in contrast to the case $F=\mathbb{B}$, in general there is no direct relation between existence and uniqueness of the minimal time projection and the subdifferentiability of the value function. Indeed, as shown in Example 4.3, even the Fréchet differentiability of $T(\cdot)$ at $\bar{x} \notin C$ does not imply the nonemptiness of the projection $\Pi_{C}^{F}(\bar{x})$ in a Hilbert space $X ; \Pi_{C}^{F}(\bar{x})$ may be not a singleton in a finite dimensions as well. Some modification of that example allows us to examine the converse situation, i.e., when $\widehat{\partial} T(\bar{x})=\emptyset$ while $\Pi_{C}^{F}(\bar{x})$ is a singleton.

Example 5.4 (existence and uniqueness of minimal time projection do not imply Fréchet subdifferentiability of minimal time function). There are subsets $C, F \subset X$ of a separable Hilbert space $X$ satisfying the standing assumptions and a point $\bar{x} \notin C$ such that $\Pi_{C}^{F}(\bar{x})$ is a singleton while $\widehat{\partial} T(\bar{x})=\emptyset$. 
Proof. Let $F$ and $C$ be given as in Example 4.3. Consider now the minimal time problem (1.1) with the same dynamics (4.3) and the modified target

$$
C_{1}:=C \cup\left\{-e_{1}\right\} .
$$

It is easy to check that $\Pi_{C_{1}}^{F}(0)=\left\{-e_{1}\right\}$, while for all $\lambda \in \mathbb{R}$ sufficiently small we compute the corresponding minimal time function by

$$
T\left(\lambda e_{1}\right)= \begin{cases}1-2 \lambda & \text { if } \lambda \geq 0 \\ 1+\lambda & \text { if } \lambda<0\end{cases}
$$

Then definition (2.8) with $\varepsilon=0$ applied to the function (5.10) gives us $\widehat{\partial} T(0)=\emptyset$.

The following simple example shows that the latter situation can occur even in $\mathbb{R}^{2}$.

Example 5.5 (existence and uniqueness of minimal time projection do not imply Fréchet subdifferentiability of minimal time function in finite dimensions). There is a nonconvex target set $C \subset \mathbb{R}^{2}$ and a convex polyhedral dynamics $F$ such that $\Pi_{C}^{F}(\bar{x})$ is a singleton at some $\bar{x} \notin C$, while $\widehat{\partial} T(\bar{x})=\emptyset$.

Proof. Let $C:=\{(x, y) \mid \min (x, y) \leq 0\}$ and $F:=\operatorname{co}\{(0,1),(1,0),(-1,-1)\}$. Then $\Pi_{C}^{F}(x, y)=\{(0,0)\}$ whenever $x=y>0$. Fixed $(a, a) \notin C$ let us denote by $\Omega$ the enlarged set $\left\{(x, y) \in \mathbb{R}^{2} \mid T_{C}^{F}(x, y) \leq T_{C}^{F}(a, a)\right\}$. It is obvious that $\Omega=(a, a)+C$ and $\widehat{N}((a, a), \Omega)=\{(0,0)\}$. By $\left[9\right.$, Theorem 3.1] we get $\widehat{\partial} T_{C}^{F}(a, a)=\emptyset$.

Theorem 5.3 gives a sufficient condition for the existence and uniqueness of the minimal time projection in terms of the target set, which is also necessary under the aforementioned subdifferentiability hypothesis. Since, as shown above, the latter property may fail in general, it would be nice to have a (weaker) necessary condition ensuring also that $\widehat{\partial} T(\bar{x}) \neq \emptyset$. Let us establish such result by assuming that the projection $\Pi_{C}^{F}(x)$ is a singleton not only at the reference point $\bar{x} \notin C$ but for each $x$ close to $\bar{x}$. Observe that the following statement is valid in general nonreflexive Asplund spaces.

Theorem 5.6 (relationships between well-posedness of minimal time projection and Fréchet differentiability of minimal time function in Asplund spaces). Let $X$ be Asplund, and let $\bar{x} \notin C$. Assume that the mapping $x \mapsto \Pi_{C}^{F}(x)$ is single-valued and continuous in a neighborhood of $\bar{x}$. If moreover the gauge $\rho_{F}(\cdot)$ is Fréchet differentiable at $\bar{y}-\bar{x}$ with $\bar{y}:=\Pi_{C}^{F}(\bar{x})$, then the minimal time function $T(\cdot)$ is also Fréchet differentiable at $\bar{x}$ and the equality $\nabla T(\bar{x})=-\nabla \rho_{F}(\bar{y}-\bar{x})$ holds.

Proof. We proceed by developing the arguments in the proof of implication (iii) $\Rightarrow$ (ii) in $[7$, Theorem 6.3] given for the case of $F=\mathbb{B}$ in Hilbert spaces. Since $\bar{\partial} T(\bar{x}) \neq \emptyset$ for the Clarke generalized gradient in Asplund spaces (see Section 2), pick $x^{*} \in \bar{\partial} T(\bar{x}) \neq \emptyset$ and denote $y:=\Pi_{C}^{F}(x)$ for all $x \in X$ close to $\bar{x}$. It is clear that $y \rightarrow \bar{y}$ when $x \rightarrow \bar{x}$. 
Then by (1.2) and (2.11) we have the relationships:

$$
\begin{aligned}
\left\langle x^{*}, \bar{y}-\bar{x}\right\rangle \leq & \limsup _{\substack{x \rightarrow \bar{x} \\
t \downarrow 0}} \frac{T(x+t(\bar{y}-\bar{x}))-T(x)}{t} \\
\leq & \limsup _{\substack{x \rightarrow \bar{x} \\
t \downarrow 0}} \frac{T(x+t(y-x))-T(x)}{t} \\
& \quad+\limsup _{\substack{x \rightarrow \bar{x} \\
t \downarrow 0}} \frac{T(x+t(\bar{y}-\bar{x}))-T(x+t(y-x))}{t} \\
\leq & \limsup _{\substack{x \rightarrow \bar{x} \\
t \downarrow 0}} \frac{\rho_{F}(y-x-t(y-x))-\rho_{F}(y-x)}{t} \\
& +\left\|F^{\circ}\right\| \lim _{x \rightarrow \bar{x}}\|\bar{y}-\bar{x}-y+x\| \\
= & -\rho_{F}(\bar{y}-\bar{x})=-\sigma_{F^{\circ}}(\bar{y}-\bar{x}),
\end{aligned}
$$

where $\left\|F^{\circ}\right\|:=\sup \left\{\left\|y^{*}\right\|\right.$ for $\left.y^{*} \in F^{\circ}\right\}$. It follows from (2.12), (2.9), and [12, Theorem 4.2] that $\bar{\partial} T(\bar{x}) \subset-F^{\circ}$. This together with (5.11) imply that $\left\langle-x^{*}, \bar{y}-\bar{x}\right\rangle=$ $\sigma_{F^{\circ}}(\bar{y}-\bar{x})$, which is equivalent to $-x^{*} \in \partial \rho_{F}(\bar{y}-\bar{x})$. The latter shows that the generalized gradient $\bar{\partial} T(\bar{x})$ is the singleton $\left\{-\nabla \rho_{F}(\bar{y}-\bar{x})\right\}$. Employing now [5, Proposition 2.2.4], we conclude that the minimal time function $T(\cdot)$ is strictly differentiable in the Hadamard sense with the strict Hadamard derivative $\nabla_{H} T(\bar{x})=-\nabla \rho_{F}(\bar{y}-\bar{x})$. It follows from the same arguments that $T(\cdot)$ is strictly Hadamard differentiable at each point $x \in X$ sufficiently close to $\bar{x}$, and that $\nabla_{H} T(x)=-\nabla \rho_{F}(y-x)$ is a unique element of the subdifferential $\partial \rho_{F}(y-x)$.

Furthermore, the Fréchet differentiability of $\rho_{F}(\cdot)$ at the point $\bar{u}:=\bar{y}-\bar{x}$ is equivalent to the Hausdorff continuity of the mapping $\partial \rho_{F}(\cdot)$ at $\bar{u}$; see, e.g., [1, Corollary 2]. Taking into account the continuity of the minimal time projection near $\bar{x}$, the (singlevalued) mapping $x \mapsto \partial \rho_{F}(y-x)$ is continuous at $\bar{x}$, which thus implies the Fréchet differentiability of $T(\cdot)$. Finally we have

$$
\nabla T(\bar{x})=\nabla_{H} T(\bar{x})=-\nabla \rho_{F}(\bar{y}-\bar{x}) \in \widehat{\partial} T(\bar{x})
$$

and complete the proof of the theorem.

Next we obtain a remarkable characterization of well-posedness of the minimal time problem (1.1) in the case of convex targets; it follows from Theorem 5.3.

Corollary 5.7 (existence and uniqueness of minimal time projection for convex targets). Let $C$ be a closed and convex subset of a reflexive space $X$, and let $\bar{x} \in X$. Then $\widehat{\partial} T(\bar{x}) \neq \emptyset$, and for any subgradient $x^{*} \in \widehat{\partial} T(\bar{x})$ the minimal time projection $\Pi_{C}^{F}(\bar{x})$ is a singleton if and only if the support function of the set $C\left(\bar{x}, x^{*}\right)$ in (5.5) is Gâteaux differentiable at $x^{*}$. 
Proof. Since the minimal time function $T(\bar{x})$ is Lipschitz continuous and convex on $X$ under the assumptions made, we get that the Fréchet subdifferential $\widehat{\partial} T(\bar{x})$ reduces to the (nonempty) subdifferential $\partial T(\bar{x})$ of convex analysis. We also have $T(\bar{x})=\widetilde{T}_{x^{*}}(\bar{x})$ for every subgradient $x^{*} \in \partial T(\bar{x})$ and thus arrive at the conclusion of the corollary by using Theorem 5.3 and the aforementioned equivalence between the single-valuedness of subdifferentials and the Gâteaux differentiability for convex functions.

Remark 5.8 (Gâteaux differentiability versus Fréchet differentiability of convex functions). Observe that, in contrast to the results of Section 4 involving the Fréchet differentiability assumption on the support function of the dynamics, we use in Theorem 5.3 and Corollary 5.7 the Gâteaux differentiability requirement on the support function of a subset of the target set. As it is well known, a Gâteaux differentiability requirement is essentially weaker than the Fréchet differentiability one for convex continuous functions in infinite dimensions. In particular, there is an equivalent norm in the space $\ell^{1}$, which is nowhere Fréchet differentiable while Gâteaux differentiable at every nonzero point; see, e.g., [18, Example 1.14(c)].

Let us next obtain another sufficient condition for well-posedness of the minimal time problem (1.1), different from the previous results of this section, that relates to the Gâteaux differentiability of the support function of an enlargement of the minimal time projection. We need the following preliminary result of its own interest; cf. [9, Theorem 4.2] for the case of Hilbert spaces.

Proposition 5.9 (minimal time function and projection estimates for convex targets in Banach spaces). Let $C \subset X$ be a closed and convex subset of a Banach space $X$. Then for any $\bar{x} \in X$ we have the inclusion

$$
\partial T(\bar{x}) \subset N(\bar{y} ; C) \text { whenever } \bar{y} \in \Pi_{C}^{F}(\bar{x}),
$$

which can be equivalently written in the dual form

$$
\Pi_{C}^{F}(\bar{x}) \subset \partial \sigma_{C}\left(x^{*}\right) \text { for each } x^{*} \in \partial T(\bar{x}) \neq \emptyset .
$$

Proof. As mentioned above, the (Lipschitz continuous) minimal time function $T(\cdot)$ is convex, and hence its Fréchet subdifferential $\widehat{\partial} T(\bar{x})$ is nonempty and reduces to the subdifferential $\partial T(\bar{x})$ of convex analysis, i.e.,

$$
T(x)-T(\bar{x}) \geq\left\langle x^{*}, x-\bar{x}\right\rangle \text { for all } x \in X \text { and } x^{*} \in \partial T(\bar{x}) .
$$

By the construction of $T(x)$ in (1.2) the latter yields, in particular, that

$$
\left\langle x^{*}, x-\bar{x}\right\rangle \leq-T(\bar{x}) \text { for all } x \in C,
$$

which in turn implies (5.12) in the case of $\bar{x} \in C$ by $T(\bar{x})=0$. If instead $\bar{x} \notin C$, we get from Corollary 3.2 that

$$
\left[\bar{y} \in \Pi_{C}^{F}(\bar{x}) \subset \bar{x}+T(\bar{x}) \mathfrak{J}_{F}\left(-x^{*}\right)\right] \Longrightarrow\left[\left\langle-x^{*}, \bar{y}-\bar{x}\right\rangle=T(\bar{x})\right] .
$$


It follows from (5.14) and (5.15) that $\left\langle x^{*}, x-\bar{y}\right\rangle \leq 0$ for all $x \in C$. Hence $x^{*} \in N(\bar{y} ; C)$ and (5.12) holds. Furthermore, from the latter inequality we get $\left\langle x^{*}, \bar{y}\right\rangle \geq \sigma_{C}\left(x^{*}\right)$. Consequently, $\left\langle x^{*}, \bar{y}\right\rangle=\sigma_{C}\left(x^{*}\right)$ by $\bar{y} \in C$. Thus $\bar{y} \in \partial \sigma_{C}\left(x^{*}\right)$, which justifies (5.13) as well as the equivalence between (5.12) and (5.13).

Now we are ready to derive the aforementioned sufficient condition for well-posedness of the minimal time problem with an arbitrary closed target set (1.1). Observe that we do not require the subdifferentiability of $T$.

Theorem 5.10 (existence and uniqueness of minimal time projection via properties of its $\varepsilon$-enlargement). Let $X$ be reflexive, and let $x \notin C$. Given any $\varepsilon>0$, denote by $S(\bar{x}, \varepsilon)$ the closed convex hull of the $\varepsilon$-approximate projection $\Pi_{C}^{F}(\bar{x}, \varepsilon)$ in (2.5) and by $T_{\varepsilon}(\cdot)$ the optimal value function in the minimal time problem with the same dynamics $F$ and with the new target set $S(\bar{x}, \varepsilon)$. Assume that for some $x^{*} \in \partial T_{\varepsilon}(\bar{x})$ the support function $\sigma_{S(\bar{x}, \varepsilon)}(\cdot)$ is Gâteaux differentiable at $x^{*}$. Then the minimal time projection $\Pi_{C}^{F}(\bar{x})$ is a singleton.

Proof. Note first that the subdifferential $\partial T_{\varepsilon}(\bar{x})$ of the convex function $T_{\varepsilon}$ is nonempty. Since the space $X$ is reflexive and since the set $S(\bar{x}, \varepsilon)$ is convex and closed, the projection $\Pi_{S(\bar{x}, \varepsilon)}^{F}(\bar{x})$ on the new target set is nonempty. Moreover, we get from Proposition 5.9 that $\Pi_{S(\bar{x}, \varepsilon)}^{F}(\bar{x}) \subset \partial \sigma_{S(\bar{x}, \varepsilon)}\left(x^{*}\right)$. The latter implies, by the Gâteaux differentiability assumption, that the set $\Pi_{S(\bar{x}, \varepsilon)}^{F}(\bar{x})$ is a singleton, i.e.,

$$
\Pi_{S(\bar{x}, \varepsilon)}^{F}(\bar{x})=\{\bar{y}\}
$$

for some $\bar{y}$. Furthermore, it follows from the Gâteaux differentiability of $\sigma_{S(\bar{x}, \varepsilon)}(\cdot)$ at $x^{*}$ that $\bar{y}$ is an exposed point (not necessarily strongly exposed) of the weakly compact set $S(\bar{x}, \varepsilon)=\overline{\mathrm{co}} \Pi_{C}^{F}(\bar{x}, \varepsilon)$ meaning that the corresponding hyperplane (4.1) touches the set $S(\bar{x}, \varepsilon)$ only at $\bar{y}$. This yields that $\bar{y} \in \Pi_{C}^{F}(\bar{x}, \varepsilon) \subset C$.

Let us next justify the inequality in

$$
\rho_{F}(\bar{y}-\bar{x})=\inf _{y \in S(\bar{x}, \varepsilon)} \rho_{F}(y-\bar{x}) \leq T(\bar{x}) .
$$

Indeed, given an arbitrary $\gamma$ satisfying $0<\gamma<\varepsilon$ and using definition (1.2), we find $y \in C$ such that $T(\bar{x}) \geq \rho_{F}(y-\bar{x})-\gamma \geq \rho_{F}(y-\bar{x})-\varepsilon$. This gives $y \in S(\bar{x}, \varepsilon)$ and thus yields (5.17). Since $\bar{y} \in C$, we get $\rho_{F}(\bar{y}-\bar{x})=T(\bar{x})$, i.e., $\bar{y} \in \Pi_{C}^{F}(\bar{x}) \neq \emptyset$. Furthermore, we have

$$
\Pi_{C}^{F}(\bar{x}) \subset \Pi_{S(\bar{x}, \varepsilon)}^{F}(\bar{x}),
$$

as we are now going to see. Indeed, obviously $\Pi_{C}^{F}(\bar{x}) \subset S(\bar{x}, \varepsilon)$ and $\rho_{F}(\widetilde{y}-\bar{x})=$ $\rho_{F}(\bar{y}-\bar{x})=T_{\varepsilon}(\bar{x})$. Combining (5.16) and (5.18) we prove the uniqueness of the minimal time projection and therefore complete the proof. 


\section{Lower Regularity of Minimal Time Function and Unique- ness of Generalized Projections}

Observe that the major results of Sections 4 and 5 involve the assumption about the Fréchet subdifferentiability of the minimal time function (1.2) at the reference point, i.e., $\widehat{\partial} T(\bar{x}) \neq \emptyset$. As mentioned above, this assumption holds if the minimal time function $T(\cdot)$ is lower regular at $\bar{x}$ and the space $X$ is either Asplund (e.g., reflexive), or $X$ is arbitrary Banach and $T(\cdot)$ is convex on $X$. On the other hand, it is well known (see $[14$, p. 111] and the references therein) that, in the case of the metric projection (1.6) of $\bar{x} \notin C$ on a closed subset $C$ of a finite-dimensional Euclidean space, i.e., for $X=\mathbb{R}^{n}$ and $F=\mathbb{B}$ in (1.4), the lower regularity of $T(\cdot)=\operatorname{dist}(\cdot ; C)$ at $\bar{x}$ is equivalent to its differentiability at this point and provides a necessary and sufficient condition for $\Pi_{C}(\bar{x})$ to be a singleton. This characterization is essentially due to the fact that the distance function is semiconcave around out-of-set points.

In this section we pursue a twofold goal. First to illustrate by examples that the aforementioned relationships are no longer true for the minimal time problem (1.1) with $F \neq \mathbb{B}$ in both finite and infinite dimensions. Then we present sufficient conditions, which ensure (and some of them are also necessary for) the lower regularity of the minimal time function (1.2) at in-set and out-of-set points.

Let us start with an example showing that, already in $X=\mathbb{R}^{2}$, the minimal time projection $\Pi_{C}^{F}(\bar{x})$ can be a singleton for some $\bar{x} \notin C$ while the minimal time function $T(\cdot)$ is not differentiable and even not lower regular at this point, although it admits a nonempty and single-valued Fréchet subdifferential.

Example 6.1 (single-valuedness of minimal time projection does not imply either differentiability or lower regularity of minimal time function in finite dimensions). There is a nonconvex target set $C \subset \mathbb{R}^{2}$ and a convex polyhedral dynamics $F$ such that $\Pi_{C}^{F}(\bar{x})$ is a singleton at some $\bar{x} \notin C$ while $T(\cdot)$ is not lower regular at $\bar{x}$.

Proof. Consider an even function $\varphi: \mathbb{R} \rightarrow \mathbb{R}$ given on $\mathbb{R}_{+}:=[0, \infty)$ by

$\varphi(x):= \begin{cases}0 & \text { for } x=0 \\ k\left(x-\frac{1}{k}\right)-\frac{1}{k^{2}} & \text { for } \frac{1}{k} \leq x \leq \frac{k^{2}+1}{k^{3}}, k \geq 2 \\ \frac{k^{3}(k-1)}{(k-1)^{2}\left(-k^{2}+k-1\right)}\left(x-\frac{1}{k-1}\right)-\frac{1}{(k-1)^{2}} & \text { for } \frac{k^{2}+1}{k^{3}} \leq x \leq \frac{1}{k-1}, k \geq 2 \\ +\infty & \text { for } x>1\end{cases}$

and define the closed target set $C \subset \mathbb{R}^{2}$ in question by the epigraph of this function

$$
C:=\left\{(x, y) \in \mathbb{R}^{2} \mid y \geq \varphi(x)\right\}
$$


depicted at Figure 1. It is easy to see that $\varphi$ is differentiable at $x=0$ with $\varphi^{\prime}(0)=0$, and thus $\widehat{N}((0,0) ; C)=(0,-1) \mathbb{R}_{+}$. Consider now the dynamics

$$
F:=\operatorname{co}\left\{\left(0, \frac{1}{2}\right),\left(\frac{1}{2},-\frac{1}{2}\right),\left(-\frac{1}{2},-\frac{1}{2}\right)\right\}
$$

and observe that $\Pi_{C}^{F}(0,-1 / 2)=\{(0,0)\}$ and that $T(0,-1 / 2)=1$. Letting further

$$
\Omega:=\left\{(x, y) \in \mathbb{R}^{2} \mid T(x, y) \leq 1\right\},
$$

we get the representation of the Fréchet normal cone $(2.13)$ to this set at $(0,-1 / 2)$ :

$$
\widehat{N}((0,-1 / 2) ; \Omega)=(0,-1) \mathbb{R}_{+} .
$$

In fact, there are no points belonging to $\Omega$ below the parabola $y=-1 / 2-x^{2}$ for all $x$ sufficiently close to 0 . On the other hand, all the points

$$
z_{k}:=\left(\frac{1}{k},-\frac{1}{k^{2}}-\frac{1}{2}\right), \quad k \geq 2,
$$

belong to both the set $\Omega$ in (6.1) and the aforementioned parabola for all $k \geq 2$. Thus it follows from $[9$, Theorem 3.1] that

$$
\widehat{\partial} T(0,-1 / 2)=\{(0,-\lambda)\}, \text { where } \lambda \in \mathbb{R} \text { is such that } \rho_{F^{0}}(0, \lambda)=1 .
$$

Observe further that for each $k \geq 2$ if the triangle $z_{k}+F$ is displaced a little in the direction $e=(1,2)$, then it continues to touch the boundary of the target $C$ at the point $\left(1 / k,-1 / k^{2}\right)$ only; in the other words

$$
\Pi_{C}^{F}\left(z_{k}+t e\right)=\left(\frac{1}{k},-\frac{1}{k^{2}}\right) \text { and } T\left(z_{k}+t e\right)=1
$$

for all $t \geq 0$ sufficiently small (see Figure 1 ). Then for each $t>0$ we have

$$
(2,-1) \in \widehat{N}\left(z_{k}+t e ; \Omega\right), \quad k \geq 2 .
$$

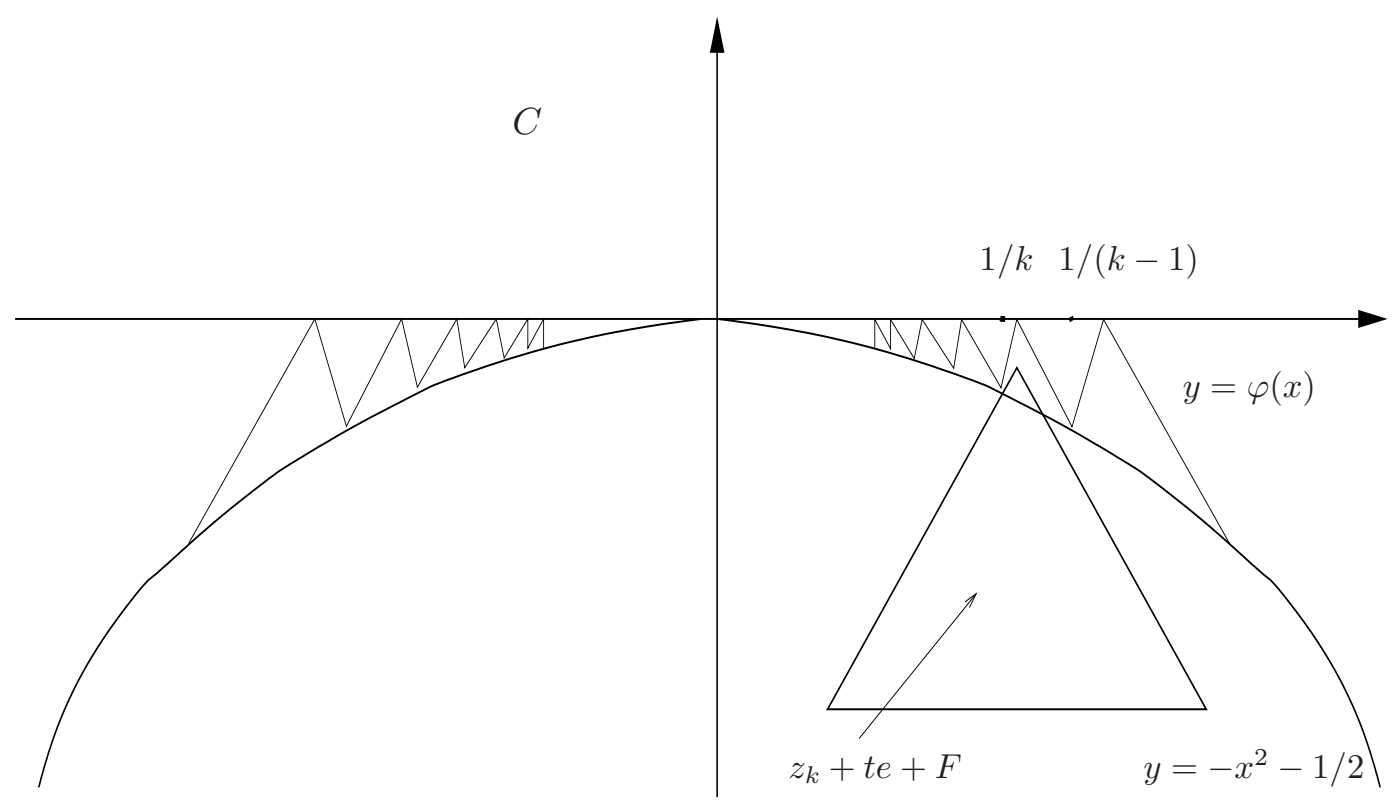


Figure 1

Thus there exists an appropriate constant $\tilde{\lambda}>0$ such that

$$
\widetilde{\lambda}(2,-1) \in \widehat{\partial} T\left(z_{k}+t e\right) \text { for small } t \geq 0 \text { and } k \geq 2 .
$$

Passing to the limit in the latter inclusions as $k \rightarrow \infty$ and $t \downarrow 0$, we arrive at

$$
\widetilde{\lambda}(2,-1) \in \partial T(0,1 / 2)
$$

for the limiting subdifferential (2.9). This implies that $\partial T(0,-1 / 2) \neq \widehat{\partial} T(0,-1 / 2)$, and hence the minimal time function $T(\cdot)$ is not lower regular at $(0,-1 / 2)$.

It is worth mentioning further that there are sets $C, F \subset \mathbb{R}^{2}$ and a point $\bar{x} \notin C$ such that the minimal time function is Fréchet subdifferentiable at $\bar{x}$ with $\widehat{\partial} T(\bar{x})$ not being a singleton, while the minimal time projection $\Pi_{C}^{F}(\bar{x})$ is a singleton. Indeed, it holds, e.g., in the case of the sets

$$
C:=\left\{\left(x_{1}, x_{2}\right) \in \mathbb{R}^{2} \mid x_{1} \leq 0, x_{2} \leq 0\right\} \text { and } F:=[-1,1] \times[-1,1]
$$

for any point $\bar{x}=\left(\bar{x}_{1}, \bar{x}_{2}\right)$ with $\bar{x}_{1}=\bar{x}_{2}>0$.

Observe also that, as shown in Example 4.3, the Fréchet differentiability of the minimal time function $T(\cdot)$ at $\bar{x} \notin C$ does not imply the nonemptiness of the minimal time projection $\Pi_{C}^{F}(\bar{x})$ in a Hilbert space $X$. Some modification of that example allows us to examine the situation when $\widehat{\partial} T(\bar{x})=\emptyset$ (and thus $T(\cdot)$ is definitely not lower regular at $\bar{x})$, while $\Pi_{C}^{F}(\bar{x})$ is a singleton.

In the rest of the section we study the lower regularity property of the minimal time function (1.2) in infinite-dimensional spaces. This topic is of its own interest as a part of well-posedness of the minimal time problem (1.1) while, as seen above, is important from the viewpoint of existence and uniqueness of the minimal time projection (1.4).

We begin by establishing a characterization of the lower regularity of $T(\cdot)$ at in-set points of target sets in arbitrary Banach spaces.

Proposition 6.2 (characterization of lower regularity of minimal time functions at in-set points of targets in Banach spaces). Let $\bar{x} \in C$ under the standing assumptions made. Then the minimal time function $T(\cdot)$ is lower regular at $\bar{x}$ if and only if the target set $C$ is normally regular at this point.

Proof. It follows from [12, Theorem 4.1] (for $F=\mathbb{B}$ it was first proved in [13]) that

$$
\widehat{\partial} T(\bar{x})=\widehat{N}(\bar{x} ; C) \cap\left\{x^{*} \in X^{*} \mid \rho_{F^{\circ}}\left(x^{*}\right) \leq 1\right\}
$$

Furthermore, we get from [17, Theorem 3.6] the representation

$$
N(\bar{x} ; C)=\bigcup_{\lambda \geq 0} \lambda \partial T(\bar{x})
$$


which was first obtained in $\left[20\right.$, Proposition 2.7] for $F=\mathbb{B}$. Since $0 \in \operatorname{int} F^{\circ}$, equality (6.2) easily implies the representation

$$
\widehat{N}(\bar{x} ; C)=\bigcup_{\lambda \geq 0} \lambda \widehat{\partial} T(\bar{x})
$$

with the convention that $0 \times \emptyset=0$. Combining (6.3) and (6.4), we arrive at the equivalence stated in the proposition.

Next we derive sufficient conditions for lower regularity of the minimal time function $T(\cdot)$ at out-of-set points in arbitrary Banach spaces. To proceed, let us recall two additional constructions needed in what follows.

Given a target set $C \subset X$ and a point $\bar{x} \notin C$, define the minimal time enlargement of $C$ relative to $\bar{x}$ by

$$
C_{r}:=\{x \in X \mid T(x) \leq r\} \text { with } r=T(\bar{x})>0 .
$$

Given further a function $\varphi: X \rightarrow \overline{\mathbb{R}}$ on a Banach space with $\varphi(\bar{x})<\infty$ and following [15], define the right-sided limiting subdifferential of $\varphi$ at $\bar{x}$ by

$$
\begin{aligned}
& \partial_{\geq} \varphi(\bar{x}):=\left\{x^{*} \in X^{*} \mid \quad \exists \text { sequences } \varepsilon_{k} \downarrow 0, x_{k} \rightarrow \bar{x} \text { and } x_{k}^{*} \stackrel{w^{*}}{\rightarrow} x^{*}\right. \\
& \text { such that } \left.\varphi\left(x_{k}\right) \downarrow \varphi(\bar{x}) \text { and } x_{k}^{*} \in \widehat{\partial}_{\varepsilon_{k}} \varphi\left(x_{k}\right)\right\} \text {. }
\end{aligned}
$$

Comparing (6.6) with the definition of the basic subdifferential (2.9), we see that the only difference between these two constructions is that $\varphi\left(x_{k}\right) \rightarrow \varphi(\bar{x})$ with $\varphi\left(x_{k}\right) \geq \varphi(\bar{x})$ in (6.6) while the latter requirement is omitted in (2.9). It immediately follows that

$$
\widehat{\partial} \varphi(\bar{x}) \subset \partial_{\geq} \varphi(\bar{x}) \subset \partial \varphi(\bar{x}) .
$$

Note that both inclusions in (6.7) are generally strict even in finite dimensions and that we can equivalently put $\varepsilon_{k} \equiv 0$ in (6.6) if $X$ is Asplund and $\varphi$ is lower semicontinuous around $\bar{x}$; see $[15,16]$ and $[14$, Subsection 1.3.3] for these and other properties of the right-sided limiting subdifferential (6.6).

Proposition 6.3 (lower regularity of minimal time function at out-of-set points in Banach spaces). Assume that the minimal time enlargement (6.5) is normally regular at $\bar{x} \notin C$. Then the minimal time function $T(\cdot)$ is lower regular at this point.

Proof. It follows from [12, Theorem 4.2] (see also [17, Theorem 4.2] for another proof and correction) that

$$
\widehat{\partial} T(\bar{x})=\widehat{N}\left(\bar{x} ; C_{r}\right) \cap\left\{x^{*} \in X^{*} \mid \rho_{F^{\circ}}\left(x^{*}\right) \leq 1\right\}
$$

in terms of the enlargement $(6.5)$; for $F=\mathbb{B}$ it was shown in $[3,13]$. On the other hand, we get from [17, Theorem 4.4] by using the right-sided subdifferential (6.6) that

$$
N\left(\bar{x} ; C_{r}\right)=\bigcup_{\lambda \geq 0} \lambda \partial_{\geq} T(\bar{x})
$$


Comparing (6.8) and (6.9) and taking (6.7) into account allow us to arrive at the conclusion of the proposition.

Our final result provides sufficient conditions of another type ensuring the lower regularity of $T(\cdot)$ at out-of-set points in terms of their generalized projection on the target set $X$ under additional assumptions in Hilbert spaces.

Recall that the proximal normal cone to a set $\Omega \subset X$ at $\bar{x} \in \Omega$ is defined by

$$
N_{p}(\bar{x} ; \Omega):=\left\{x^{*} \in X^{*} \mid \exists \gamma>0 \text { with }\left\langle x^{*}, x-\bar{x}\right\rangle \leq \gamma\|x-\bar{x}\|^{2} \text { for all } x \in \Omega\right\} .
$$

We always have the inclusions

$$
N_{p}(\bar{x} ; \Omega) \subset \widehat{N}(\bar{x} ; \Omega) \subset N(\bar{x} ; \Omega), \quad \bar{x} \in \Omega,
$$

both of which can be strict already in finite dimensions. Accordingly, a set $\Omega$ is proximally regular at a point $\bar{x} \in \Omega$ if

$$
N_{p}(\bar{x} ; \Omega)=N(\bar{x} ; \Omega) .
$$

It follows directly from (6.11) that the proximal regularity of a set implies its normal regularity at the corresponding point.

Now we are ready to derive the aforementioned sufficient conditions for lower regularity of the minimal time function, and give moreover a precise representation of its subdifferential(s) under consideration.

Theorem 6.4 (lower regularity of minimal time function at out-of-set points in Hilbert spaces). In the framework of problem (1.1) let the space $X$ be Hilbert, and let $\bar{x} \notin C$. Assume furthermore that the minimal time projection $\Pi_{C}^{F}(\cdot)$ is single-valued around $\bar{x}$ and satisfies the following "one-point" Hölder property at $\bar{x}$ : there are constants $K>0,1 / 2<\alpha \leq 1$, and neighborhood $U$ of $\bar{x}$ such that

$$
\left\|\Pi_{C}^{F}(x)-\Pi_{C}^{F}(\bar{x})\right\| \leq K\|x-\bar{x}\|^{\alpha} \text { for all } x \in U .
$$

If the set $C$ is proximally regular around $\bar{y}:=\Pi_{C}^{F}(\bar{x})$, then the minimal time function $T(\cdot)$ is lower regular at $\bar{x}$, and we have the representation

$$
\partial T(\bar{x})=N(\bar{y} ; C) \cap\left(-\partial \rho_{F}(\bar{y}-\bar{x})\right) .
$$

Proof. It follows from [9, Theorem 3.3] and the proximal regularity of $C$ around $\bar{y}$ that

$$
\widehat{\partial} T(x) \subset N_{p}(y ; C) \cap\left(-\partial \rho_{F}(y-x)\right) \text { for } y=\Pi_{C}^{F}(x) \text { and any } x \in U,
$$

where $U$ is taken from the condition (6.12). Let us now justify the "one-point" version

$$
N_{p}(\bar{y} ; C) \cap\left(-\partial \rho_{F}(\bar{y}-\bar{x})\right) \subset \widehat{\partial} T(\bar{x})
$$

of the opposite inclusion to (6.14) under all the assumptions made. Picking $x^{*}$ from the set on the left-hand side of (6.15), we get by the subdifferential construction of convex analysis that the inclusion $-x^{*} \in \partial \rho_{F}(\bar{y}-\bar{x})$ reads as

$$
\rho_{F}(u) \geq \rho_{F}(\bar{y}-\bar{x})+\left\langle-x^{*}, u-\bar{y}+\bar{x}\right\rangle \text { for all } u \in X .
$$


Furthermore, that of $x^{*} \in N_{p}(\bar{y} ; C)$ signifies by (6.10) the existence of $\gamma>0$ with

$$
\left\langle x^{*}, v-\bar{y}\right\rangle \leq \gamma\|v-\bar{y}\|^{2} \text { for all } v \in C .
$$

Putting then $u=y-x$ for $x \in U$ and $y=\Pi_{C}^{F}(x)$, we get from (6.16) the estimate

$$
\rho_{F}(y-x)-\rho_{F}(\bar{y}-\bar{x})-\left\langle x^{*}, x-\bar{x}\right\rangle \geq\left\langle-x^{*}, y-\bar{y}\right\rangle .
$$

Combining (6.17) and (6.18) allows us to conclude that

$$
\begin{aligned}
& \liminf _{x \rightarrow \bar{x}} \frac{T(x)-T(\bar{x})-\left\langle x^{*}, x-\bar{x}\right\rangle}{\|x-\bar{x}\|} \geq \liminf _{x \rightarrow \bar{x}} \frac{\left\langle-x^{*}, y-\bar{y}\right\rangle}{\|x-\bar{x}\|} \\
& \geq \liminf _{x \rightarrow \bar{x}} \frac{-\gamma\|y-\bar{y}\|^{2}}{\|x-\bar{x}\|} \geq-K \gamma \lim _{x \rightarrow \bar{x}}\|x-\bar{x}\|^{2 \alpha-1}=0,
\end{aligned}
$$

where the latter inequality is due to the Hölder condition (6.12). By definition (2.8) with $\varepsilon=0$, we get from (6.19) that $x^{*} \in \widehat{\partial} T(\bar{x})$ and thus justify inclusion (6.15).

Using next construction (2.9) of the basic subdifferential in the case of Hilbert spaces and then inclusion (6.14) and the first one in (6.11) gives us the relationships

$$
\begin{aligned}
\partial T(\bar{x})=w-\operatorname{Limsup}_{x \rightarrow \bar{x}} \widehat{\partial} T(x) & \subset w-\operatorname{Limsup}_{x \rightarrow \bar{x}}\left[N_{p}(y ; C) \cap\left(-\partial \rho_{F}(y-x)\right)\right] \\
& \subset w-\operatorname{Limsup}_{x \rightarrow \bar{x}}\left[\widehat{N}(y ; C) \cap\left(-\partial \rho_{F}(y-x)\right)\right],
\end{aligned}
$$

where clearly $y=\Pi_{C}^{F}(x) \rightarrow \bar{y}$ as $x \rightarrow \bar{x}$. It follows further from the normal cone definitions (2.13) and the graph closedness of the subdifferential of convex analysis that the right-hand side of the last inclusion in (6.20) reduces to $N(\bar{y} ; C) \cap\left(-\partial \rho_{F}(\bar{y}-\bar{x})\right)$. This yields by inclusions (6.15), (2.10), and the proximal regularity of $C$ at $\bar{y}$ that

$$
N(\bar{y} ; C) \cap\left(-\partial \rho_{F}(\bar{y}-\bar{x})\right)=N_{p}(\bar{y} ; C) \cap\left(-\partial \rho_{F}(\bar{y}-\bar{x})\right) \subset \widehat{\partial} T(\bar{x}) \subset \partial T(\bar{x}) .
$$

Combining (6.20) and (6.21), we get the lower regularity of $T$ at $\bar{x}$ and thus complete the proof of theorem.

Note that some verifiable sufficient conditions ensuring the Hölder property of the minimal time projection (6.12) appear in [11].

Acknowledgement. The authors thank two anonymous referees and Lionel Thibault for their sharp remarks that allowed us to improve the original presentation.

\section{References}

[1] Asplund, E., Rockafellar, R.T.: Gradients of convex functions. Trans. Amer. Math. Soc. 139, 443-467 (1969)

[2] Borwein, J.M., Giles, J.R.: The proximal normal formula in Banach spaces. Trans. Amer. Math. Soc. 302, 371-381 (1987) 
[3] Bounkhel, M., Thibault, L.: On various notions of regularity of sets in nonsmooth analysis. Nonlinear Anal. 48, 223-246 (2002)

[4] Canino, A: On p-convex sets and geodesics. J. Diff. Equat. 75, 118-157 (1988)

[5] Clarke, F.H.: Optimization and Nonsmooth Analysis. Wiley-Interscience, New York (1983)

[6] Clarke, F.H., Stern, R.J., Wolenski, P.R.: Proximal smoothness and the lower $C^{2}$ property. J. Convex Analysis 2, 117-144 (1995)

[7] Colombo, G., Goncharov, V.V.: Variational inequalities and regularity properties of closed sets in Hilbert spaces. J. Convex Anal. 8, 197-221 (2001)

[8] Colombo, G., Wolenski, P.R.: The subgradient formula for the minimal time function in the case of constant dynamics in Hilbert space. J. Glob. Optim. 28, 269-282 (2004)

[9] Colombo, G., Wolenski, P.R.: Variational analysis for a class of minimal time functions in Hilbert spaces. J. Convex Anal. 11, 335-361 (2004)

[10] Goncharov, V.V., Pereira, F.: Neighbourhood retractions of nonconvex sets in a Hilbert space via sublinear functionals. J. Convex Anal. 18 (2011)

[11] Goncharov, V.V., Pereira, F.: Geometric conditions for regularity in a minimum time problem with constant dynamics, in preparation

[12] He, Y., Ng, K.F.: Subdifferentials of a minimum time function in Banach spaces. J. Math. Anal. Appl. 321, 896-910 (2006)

[13] Kruger, A.Y.: $\varepsilon$-Semidifferentials and $\varepsilon$-normal elements. Depon. VINITI, No. 133181, Moscow (1981)

[14] Mordukhovich, B.S.: Variational Analysis and Generalized Differentiation, I: Basic Theory. Grundlehren Series (Fundamental Principles of Mathematical Science), vol. 330. Springer, Berlin (2006)

[15] Mordukhovich, B.S., Nam, N.M.: Subgradients of distance functions with applications to Lipschitzian stability. Math. Program. 104, 635-668 (2005)

[16] Mordukhovich, B.S., Nam, N.M.: Subgradients of distance functions at out-of-set points. Taiwanese J. Math. 10, 299-326 (2006)

[17] Mordukhovich, B.S., Nam, N.M.: Limiting subgradients of minimal time functions in Banach spaces. J. Global Optim. 46, 615-633 (2010)

[18] Phelps, R.R.: Convex Functions, Monotone Operators and Differentiability. Lecture Notes Math., vol. 1364. Springer, New York (1989) 
[19] Poliquin, R.A., Rockafellar, R.T., Thibault, L.: Local differentiability of distance functions. Trans. Amer. Math. Soc. 352, 5231-5249 (2000)

[20] Thibault, L.: On subdifferentials of optimal value functions. SIAM J. Control Optim. 29, 1019-1036 (1991) 